\title{
INFORMACÕES CONTIDAS NOS MANUAIS DE TESTES DE INTELIGÊNCIA PUBLICADOS NO BRASIL
}

\author{
Ana Paula Porto Noronha \\ Fernanda Argentini Sartori \\ Fernanda Andrade Freitas \\ Fernanda Ottati
}

\begin{abstract}
RESUMO. O objetivo do presente estudo foi analisar a presença de informações relativas aos dados de identificação e aos dados de aplicação e de avaliação, nos manuais dos testes psicológicos. Foram verificados vinte e um testes de inteligência. Cada instrumento foi submetido a uma análise individual, com base em critérios de um questionário para avaliar a qualidade dos testes usados na Espanha (Prieto \& Muñiz, 2000). Os resultados revelaram que alguns testes não possuem, nos seus manuais, informações como data de publicação, nome do autor ou bibliografia.
\end{abstract}

Palavras-chaves: construção de testes, testes de inteligência, manual de teste.

\section{DATA IN TEST MANUALS: A STUDY OF IQ TESTS PUBLISHED IN BRAZIL}

\begin{abstract}
This paper analyses information concerning identification, application and evaluation data in psychological test manuals. Twenty IQ tests were analyzed. Each test was analyzed separately according to the criteria of a questionnaire used to evaluate the quality of tests used in Spain. Results showed that some test manuals do not provide the date of publishing, the name of the author or the bibliography.
\end{abstract}

Key words: test constuction, intelligence tests, test manual.

\section{INTRODUÇÃO}

Os testes psicológicos são instrumentos úteis para a realização da avaliação psicológica, tendo-se em vista que oferecem uma "medida objetiva e padronizada de uma amostra do comportamento" (Anastasi \& Urbina, 2000, p. 18). No Brasil, eles são exclusivos dos profissionais de psicologia e não têm apresentado um desenvolvimento satisfatório, pois são muitos os problemas encontrados nesta área de conhecimento, tais como, instrumentos desatualizados e sem estudos nacionais, poucos instrumentos para avaliar determinados construtos, má utilização do instrumental por parte dos profissionais, dentre tantas outras dificuldades.
Segundo Almeida (1999), ao contrário do crescimento encontrado em outros campos, como informática, saúde e telecomunicações, os testes permanecem imutáveis; o autor acredita que os testes utilizados estejam próximos dos criados no começo do século.

Algumas iniciativas têm sido tomadas no sentido de se resgatar a legitimidade dos instrumentos padronizados. Os esforços não têm sido poucos e um destaque deve ser dado ao trabalho de Wechsler (1999), que organizou um Guia de Procedimentos Éticos para a Avaliação Psicológica, no qual estão incluídas as informações gerais sobre os princípios, uso, seleção, aplicação, correção e interpretação dos testes psicológicos.

Nesta mesma perspectiva, outras iniciativas, de âmbito internacional, têm sido acrescentadas, como as Diretrizes Internacionais para o Uso dos Testes,

\footnotetext{
Doutora em Psicologia: ciência e profissão pela PUC-Campinas; Docente do Curso de Psicologia e de Estudos Pós-graduados em Psicologia da Universidade São Francisco / campus Itatiba-SP.

Endereço para correspondência: Rua Francisco Pinto Osório, 40, Jardim Morumbi, CEP 13.250-000, Itatiba- São Paulo. Email: noronhafagundes@netwave.com.br ou ananoronha@saofrancisco.edu.com

\# Aluna do Curso de Psicologia da Universidade São Francisco / campus Itatiba.

II Bolsista de Iniciação Científica
} 
elaboradas pela Comissão Internacional de Testes (ITC, 2001). Neste trabalho são apresentadas as diretrizes gerais para o uso dos testes, no que se refere à escolha e aos princípios de aplicação, avaliação e interpretação. Além disto, o trabalho versa sobre o estabelecimento de políticas sobre o uso dos testes.

Uma publicação conhecida mundialmente é o Mental Measurements Yearbook (anuário de medidas mentais) do Buros Institute of Mental Measurements, que examina continuamente os testes psicológicos. Ele oferece, além da listagem completa de instrumentos de avaliação, uma breve descrição de cada um deles. Cunha (2000) também preparou uma listagem de testes com algumas informações sobre cada instrumento e comentários gerais. Vale ressaltar que, embora a autora não aborde todos os instrumentos psicológicos publicados no Brasil, o esforço é louvável, uma vez que se caminha para o desenvolvimento de materiais que abordem a avaliação da qualidade das técnicas padronizadas.

Este tipo de trabalho é freqüente na literatura internacional; não raras vezes são publicados artigos que se referem à avaliação da precisão e da validade de instrumentos ou que versam sobre a preocupação com a padronização deles (Geisinger, 2000; Silverstein \& Nelson, 2000; Adams, 2000; Okazaki \& Sue, 2000). Sabe-se que esta linha de pesquisa não é simples (Strauss, Spreen \& Hunter, 2000), mas não há dúvidas de que é imprescindível para o desenvolvimento da avaliação psicológica.

Segundo Salvia e Ysseldyke (1991), o psicólogo deve estudar cuidadosamente os manuais de testes e, segundo determinação do comitê da American Psychology Association, da American Educational Research Association e do National Council on Measurement in Education, qualquer manual deve oferecer ao usuário as informações importantes para que a escolha do material esteja em concordância com os objetivos e necessidades da medida.

Ainda no que diz respeito ao trabalho que vem sendo desenvolvido pela Comissão Internacional de Testes, nas Diretrizes para Adaptação/Tradução dos Testes, consta que os construtores e os aplicadores de testes deveriam tratar de prevenir os diferentes tipos de problemas e tomar medidas oportunas para evitálos, mediante a preparação de manuais adequados.

$\mathrm{Na}$ prática profissional brasileira o que se encontra é o descuido em relação a grande parte dos instrumentos comercializados, que não apresentam a qualidade técnica necessária e indispensável para o exercício profissional adequado. Para Sisto, Sbardelini e Primi (2001) tal quadro parece estar sendo revertido, considerando-se que o Conselho Assessor de Psicologia no CNPq definiu a subárea de "Fundamentos e Medidas em Psicologia" como uma das cinco, dentre dez existentes, que mereceriam atenção e investimentos.

Por outro lado, as dificuldades presentes na realização da avaliação psicológica não giram exclusivamente em torno de manuais incompletos, mas dizem respeito sobretudo, ao instrumento como um todo, às suas limitações, às dimensões avaliadas e à falta de compreensão do psicólogo sobre a contribuição limitada que o teste pode oferecer. Tendo-se em vista que ele deverá ser utilizado apenas como um auxílio, cabe ao psicólogo compreender as demais questões conceituais e teóricas decorrentes deste uso (Sbardelini, 2001).

Considerando a urgente necessidade de realização de pesquisas na área de avaliação psicológica, o presente estudo objetivou analisar os manuais de testes psicológicos de inteligência, em relação às seguintes informações: dados gerais de identificação (nome, autor, data de publicação e editor), dados de aplicação e de avaliação (variável medida, áreas de aplicação, formato dos itens, número de itens, suporte, correção, transformação da pontuação, escalas utilizadas, tempo de aplicação, documentação oferecida e bibliografia) e características psicométricas (validade e precisão).

\section{MÉTODO}

\section{Instrumentos consultados}

Os instrumentos utilizados na realização da presente pesquisa foram 21 testes psicológicos de avaliação da inteligência, que possuíam manual em português, a saber: Lorge, I.; Blue, L.; Burgemeister, B. (1993) Escala de Maturidade Mental - Columbia. Casa do Psicólogo; Wechsler David. (1964) Escala Wechsler de Avaliação da Inteligência para Crianças - WISC. Cepa; Wechsler, Solange Muglia. (s/d) $\mathrm{O}$ Desenho da Figura Humana DFH. Livro Pleno; D-70. (1988) Editions du Centre de Psychologie Appliqueé: Casa do Psicólogo; Pichot, Pierre. (s/d) D-48. Cepa; Técnicos do ISOP. (s/d) Teste Barcelona. Cepa; Raven, J. (1977, s/d) Teste de Matrizes Progressivas - escalas geral e avançada. Cepa; Raven, J. C.; Raven, J.; Court, J. H. (1988) Matrizes Progressivas Coloridas. Cetepp; Boccalandro, Efraim Rojas. (1979) Teste de Inteligência Não-verbal - G-38. Vetor; Wechsler, David. (s/d) Teste Coletivo de Inteligência para Adultos CIA forma I. Cepa; (Boccalandro, Efraim Rojas. (1966) Teste Não-verbal de inteligência - G36. Vetor; Maia, 
Jacyr. (s/d) Prova de Nível Mental. Cepa; Cattell, R. B. \& Cattell, A. K. S. (s/d) Teste Eqüicultural de Inteligência escalas 2 e 3. Cepa; Oliveira, Rynaldo de. (s/d) Teste Não-verbal de Inteligência - R1. Vetor; Weil, Pierre \& Nick, Eva. (s/d) Teste de Inteligência Não Verbal - INV. Cepa; Souza, Fábio de Vasconcellos \& Suzy Vijande Cambrais. (s/d) Teste dos Relógios. Vetor; Del Nero, Carlos. (1982) Teste de Capacidades Intelectuais. Vetor; (Cunha, J. A.; Moraes, M. J. B.; Rocha, N. S. R.; Werba, L.; Valle, R.; Moraes, J. E. B.; Salermo, J. C.; Costa, L. (s/d) Teste de Sondagem Intelectual. Cepa; Boccalandro, Efraim Rojas. (1978) Teste Verbal de Inteligência - V-47. Vetor e Cubos de Kohs. (s/d) Cepa.

Vale destacar que as datas que aparecem entre parênteses são as dos manuais avaliados, e não necessariamente as datas originais dos instrumentos ou das respectivas adaptações, traduções ou padronizações.

\section{MATERIAL}

Para a viabilização da pesquisa as autoras se basearam no questionário proposto por Prieto \& Muñiz (2000), originalmente criado para avaliar a qualidade dos testes usados na Espanha. O instrumento aborda os seguintes aspectos apresentados nos manuais dos testes psicológicos: descrição das variáveis medidas (obejtivos da medida); áreas de aplicação (indicações para o uso do instrumento); formato (resposta livre, resposta dicotômica, eleição múltipla, Likert, outros) e número de itens; suporte (administração oral, papel e lápis, manipulação de materiais, informatizado e outros); procedimentos de correção (crivo, leitura ótica, manual, com a ajuda de especialistas, outros); pontuação (descrição do procedimento de obtenção da pontuação); escalas utilizadas (percentil, QI, T, outros); tempo para administração; preço; qualidade do material (objetos, materiais impressos ou software); qualidade da documentação (manual, livros, folha de resposta, caderno); fundamentação teórica (existência e clareza da fundamentação do instrumento); referências bibliográficas (se constam referências para consulta no manual); adaptação do teste (se o teste foi traduzido, adaptado ou padronizado para a realidade brasileira); qualidade das instruções (claras e precisas, adequadas para a população na qual o teste será aplicado) e dos itens (aspectos formais como redação e desenhos); facilidade para compreender a tarefa e para registrar respostas; análise dos itens; validade e fidedignidade e normas.

Vale ressaltar que este estudo trabalhou com as informações contidas nos respectivos manuais dos testes, e os itens avaliados foram: nome do teste, autor, editor, data da publicação, variável (classificação geral da variável que se pretende medir), áreas de aplicação (indicações para o uso), formato dos itens (resposta livre, resposta dicotômica, eleição múltipla, tipo Likert, adjetivos bipolares, outros), número de itens, suporte (administração oral, lápis e papel, manipulativo, informatizado, outros), correção (crivo, leitura ótica, realizada por aplicador, com a ajuda de expertos e outros), transformação da pontuação (característica não aplicável ao instrumento, normatizada, não normatizada), escalas utilizadas (percentil, QI, T, outros), tempo de aplicação, documentação oferecida (manual, livros ou artigos complementares, disquetes, $\mathrm{CD}$, outros), bibliografia básica acerca do teste, validade (preditiva, de construto e de conteúdo) e precisão.

\section{PROCEDIMENTO}

Após a seleção dos instrumentos que fizeram parte do presente estudo, eles foram avaliados individualmente, a partir dos critérios estabelecidos. Cada teste tinha uma ficha em que constavam os elementos de análise, a fim de facilitar a coleta. Em seguida, iniciou-se o tratamento dos dados.

\section{RESULTADOS}

Os resultados obtidos através da análise dos manuais dos instrumentos psicológicos avaliados foram classificados em dois grandes grupos, a saber: identificação do teste e dados de aplicação e de avaliação. Os dados foram organizados de modo a atender aos objetivos do estudo e foram apresentados em quatro tabelas, sendo que o critério de avaliação dos testes foi: presença (1) ou ausência (0) dos aspectos avaliados.

A Tabela 1 apresenta os resultados referentes às informações que se destinam à identificação do material consultado, como por exemplo, nome, autor, editor original e data. A nota máxima que cada instrumento poderia receber nestes aspectos era quatro.

A maioria dos testes analisados obteve a pontuação máxima dos 21 instrumentos estudados, 52,4\% (N=11) receberam a pontuação máxima. São eles: Colúmbia, WISC, Matrizes Progressivas (escala geral e escala avançada), D-48, G-38, G-36, Prova de Nível Mental, Teste dos Relógios, TCI e V-47; 33,3\% receberam a nota $3(\mathrm{~N}=7)$ : Matrizes Coloridas, Teste Eqüiculural, R1, INV, Sondagem Intelectual, Desenho da Figura Humana (D.F.H.) e D 70; e 14,3\% tiveram a nota 2 $(\mathrm{N}=3)$ : CIA, Barcelona e Cubos de Khos. 
Tabela 1. Identificação do teste.

\begin{tabular}{|c|c|c|c|c|c|}
\hline Nome teste & Nome & $\begin{array}{c}\text { Autor } \\
\text { original }\end{array}$ & $\begin{array}{c}\text { Editor } \\
\text { original }\end{array}$ & Data & Total \\
\hline Colúmbia & 1 & 1 & 1 & 1 & 4 \\
\hline WISC & 1 & 1 & 1 & 1 & 4 \\
\hline Matrizes Progressivas geral & 1 & 1 & 1 & 1 & 4 \\
\hline Matrizes Coloridas & 1 & 1 & 0 & 1 & 3 \\
\hline Matrizes Progressivas avanç. & 1 & 1 & 1 & 1 & 4 \\
\hline G-38 & 1 & 1 & 1 & 1 & 4 \\
\hline CIA- forma I & 1 & 1 & 0 & 0 & 2 \\
\hline D-48 & 1 & 1 & 1 & 1 & 4 \\
\hline G-36 & 1 & 1 & 1 & 1 & 4 \\
\hline D- 70 & 1 & 0 & 1 & 1 & 3 \\
\hline Prova de Nível Mental & 1 & 1 & 1 & 1 & 4 \\
\hline Equicultural & 1 & 1 & 1 & 0 & 3 \\
\hline $\mathrm{R} 1$ & 1 & 1 & 1 & 0 & 3 \\
\hline INV & 1 & 1 & 1 & 0 & 3 \\
\hline Teste dos Relógios & 1 & 1 & 1 & 1 & 4 \\
\hline TCI & 1 & 1 & 1 & 1 & 4 \\
\hline Sondagem Intelectual & 1 & 1 & 1 & 0 & 3 \\
\hline V 47 & 1 & 1 & 1 & 1 & 4 \\
\hline D.F.H. & 1 & 1 & 1 & 0 & 3 \\
\hline Barcelona & 1 & 1 & 0 & 0 & 2 \\
\hline Cubos de Kohs & 1 & 1 & 0 & 0 & 2 \\
\hline Total & 21 & 20 & 17 & 13 & \\
\hline
\end{tabular}

Em relação aos aspectos de identificação avaliados, o nome do teste apareceu em $100 \%$ dos instrumentos; o nome do autor apareceu em 95,2\% $(\mathrm{N}=20)$; o editor esteve presente em $80,9 \%(\mathrm{~N}=17)$; e a data da publicação do material, em $61,9 \%(\mathrm{~N}=13)$.

A Tabela 2 apresenta os dados de aplicação e de avaliação dos instrumentos. Os aspectos avaliados são: variáveis medidas, forma de aplicação, formato dos itens, número de itens, suporte, correção, pontuação, escala utilizada, tempo de aplicação, documentação aportada e referências bibliográficas que constam do manual. A pontuação máxima nesta avaliação é onze.

Dos instrumentos estudados, $57,1 \% \quad(\mathrm{~N}=12)$ receberam a pontuação máxima; $14,3 \%$ receberam nota $10 \quad(\mathrm{~N}=3) ; 23,8 \%$ obtiveram $9 \quad(\mathrm{~N}=5)$. Os instrumentos menos pontuados foram TCI, Sondagem Intelectual, V-47, Barcelona e R1.

Tabela 2. Dados de aplicação e de avaliação.

\begin{tabular}{|c|c|c|c|c|c|c|c|c|c|c|c|c|}
\hline Nome teste & Variável & Aplic. & Itens forma & $n^{\circ}$ de ítens & Suporte & Correç & Pontos & Escala & Tempo & Doct & Bibliog. & Total \\
\hline Colúmbia & 1 & 1 & 1 & 1 & 1 & 1 & 1 & 1 & 1 & 1 & 1 & 11 \\
\hline WISC & 1 & 1 & 1 & 1 & 1 & 1 & 1 & 1 & 1 & 1 & 1 & 11 \\
\hline Matriz Progres geral & 1 & 1 & 1 & 1 & 1 & 1 & 1 & 1 & 1 & 1 & 1 & 11 \\
\hline Matrizes Coloridas & 1 & 1 & 1 & 1 & 1 & 1 & 1 & 1 & 1 & 1 & 1 & 11 \\
\hline Matriz Progres avç & 1 & 1 & 1 & 1 & 1 & 1 & 1 & 1 & 1 & 1 & 1 & 11 \\
\hline G-38 & 1 & 1 & 1 & 1 & 1 & 1 & 1 & 1 & 1 & 1 & 1 & 11 \\
\hline CIA- forma I & 1 & 0 & 1 & 1 & 1 & 1 & 1 & 1 & 1 & 1 & 1 & 10 \\
\hline D-48 & 1 & 1 & 1 & 1 & 1 & 1 & 1 & 1 & 1 & 1 & 1 & 11 \\
\hline G-36 & 1 & 1 & 1 & 1 & 1 & 1 & 1 & 1 & 1 & 1 & 1 & 11 \\
\hline D- 70 & 1 & 1 & 1 & 1 & 1 & 1 & 1 & 1 & 1 & 1 & 1 & 11 \\
\hline Nível mental & 1 & 1 & 1 & 1 & 1 & 1 & 1 & 1 & 1 & 1 & 0 & $\backslash 10$ \\
\hline Equicultural & 1 & 1 & 1 & 1 & 1 & 1 & 1 & 1 & 1 & 1 & 1 & 11 \\
\hline $\mathrm{R} 1$ & 1 & 1 & 1 & 1 & 1 & 1 & 0 & 1 & 1 & 1 & 0 & 9 \\
\hline INV & 1 & 1 & 1 & 1 & 1 & 1 & 1 & 1 & 1 & 1 & 1 & 11 \\
\hline Teste dos relógios & 1 & 1 & 1 & 1 & 1 & 1 & 1 & 1 & 1 & 1 & 0 & 10 \\
\hline TCI & 1 & 0 & 1 & 1 & 1 & 1 & 1 & 1 & 1 & 1 & 0 & 9 \\
\hline Sond Intelectual & 1 & 0 & 1 & 1 & 1 & 1 & 1 & 1 & 1 & 1 & 0 & 9 \\
\hline V 47 & 1 & 0 & 1 & 1 & 1 & 1 & 1 & 1 & 1 & 1 & 0 & 9 \\
\hline D.F.H. & 1 & 1 & 1 & 1 & 1 & 1 & 1 & 1 & 1 & 1 & 1 & 11 \\
\hline Barcelona & 1 & 0 & 1 & 1 & 1 & 1 & 1 & 1 & 1 & 1 & 0 & 9 \\
\hline Cubos de Kohs & 1 & 1 & 1 & 1 & 1 & 1 & 1 & 1 & 1 & 1 & 1 & 11 \\
\hline Total & 21 & 16 & 21 & 21 & 21 & 21 & 19 & 21 & 21 & 21 & 14 & \\
\hline
\end{tabular}

Em relação aos aspectos de aplicação e de avaliação observados, alguns deles estiveram presentes em todos os testes analisados, tais como a variável medida, o formato dos itens, o número de itens, o suporte, a forma de correção, a escala, o tempo e a documentação fornecida. A bibliografia foi o elemento menos freqüente nos instrumentos.

Em relação às características psicométricas dos instrumentos, os dados podem ser encontrados na
Tabela 3. Foram avaliadas as presenças ou ausências de dados sobre validade e precisão dos testes estudados, sem que tenha sido feita uma avaliação mais criteriosa a respeito da adequação ou inadequação dos estudos desenvolvidos. Neste momento, verificou-se apenas se havia algum dado que indicasse a preocupação com as qualidades psicométricas.

Os resultados indicaram que dos testes referenciados, $14,27 \%(\mathrm{~N}=3)$ não apresentaram nenhum estudo de 
validade e precisão; são eles: Teste dos Relógios, TCI e Teste Barcelona. Enquanto 66,7\% (N=14) apresentaram os estudos destacados e em 19,0\% ( $\mathrm{N}=4)$ dos testes foram realizados um outro estudo.

Tabela 3. Qualidades psicométricas dos instrumentos.

\begin{tabular}{lccc}
\hline Nome do Teste & Validade & precisão & total \\
\hline Colúmbia & 1 & 1 & 2 \\
WISC & 1 & 1 & 2 \\
Matrizes Progressivas geral & 1 & 1 & 2 \\
Matrizes Coloridas & 0 & 1 & 1 \\
Matrizes Progressivas avanç. & 1 & 1 & 2 \\
G-38 & 1 & 1 & 2 \\
CIA- forma I & 0 & 1 & 1 \\
D-48 & 1 & 1 & 2 \\
G-36 & 1 & 1 & 2 \\
D- 70 & 1 & 1 & 2 \\
Prova de Nível Mental & 1 & 0 & 1 \\
Equicultural & 1 & 1 & 2 \\
R1 & 1 & 1 & 2 \\
INV & 1 & 1 & 2 \\
Teste dos Relógios & 0 & 0 & 0 \\
TCI & 0 & 0 & 0 \\
Sondagem Intelectual & 1 & 1 & 2 \\
V 47 & 1 & 1 & 2 \\
D.F.H. & 1 & 1 & 2 \\
Barcelona & 0 & 0 & 0 \\
Cubos de Kohs & 1 & 0 & 1 \\
\hline Total & 16 & 16 & 32 \\
\hline
\end{tabular}

A Tabela 4 apresenta a nota geral dos instrumentos. Considerando-se que o máximo de pontos que um instrumento poderia receber é 17, $33,3 \%(\mathrm{~N}=7)$ tiveram a pontuação máxima, são eles: Columbia, WISC, Matrizes Progressivas geral e avançada, G38, D48 e G36. O instrumento que teve menor nota foi o Teste Barcelona (11 pontos).

Tabela 4. Nota geral dos instrumentos nos aspectos avaliados.

\begin{tabular}{lcccc}
\hline Nome teste & Identificação Aplic/aval. Qualidade & Total \\
\hline Colúmbia & 4 & 11 & 2 & 17 \\
WISC & 4 & 11 & 2 & 17 \\
Matrizes Progressivas geral & 4 & 11 & 2 & 17 \\
Matrizes Coloridas & 3 & 11 & 1 & 15 \\
Matrizes Progressivas avanç. & 4 & 11 & 2 & 17 \\
G-38 & 4 & 11 & 2 & 17 \\
CIA- forma I & 2 & 10 & 1 & 13 \\
D-48 & 4 & 11 & 2 & 17 \\
G-36 & 4 & 11 & 2 & 17 \\
D- 70 & 3 & 11 & 2 & 16 \\
Prova de Nível Mental & 4 & 10 & 1 & 15 \\
Equicultural & 3 & 11 & 2 & 16 \\
R1 & 3 & 9 & 2 & 14 \\
INV & 3 & 11 & 2 & 16 \\
Teste dos Relógios & 4 & 10 & 0 & 14 \\
TCI & 4 & 9 & 0 & 13 \\
Sondagem Intelectual & 3 & 9 & 2 & 14 \\
V 47 & 4 & 9 & 2 & 15 \\
D.F.H. & 3 & 11 & 2 & 16 \\
Barcelona & 2 & 9 & 0 & 11 \\
Cubos de Kohs & 2 & 11 & 1 & 14 \\
Média & & & 1,52 & \\
\hline
\end{tabular}

\section{$\underline{\mathrm{DP}}$}

Para a análise estatística foi utilizado o teste de qui-quadrado para verificar a homogeneidade da distribuição dos aspectos avaliados nos testes (Xc= 31,410; n.g.1. 20; n. sign. 0,05). Houve correlações de 3,2398 para os dados de identificação, de 1,4409 para os dados de aplicação e de avaliação e 2,80184 para a qualidade psicométrica; portanto, não significativas, o que significa dizer que os itens avaliados se distribuem igualmente nos instrumentos estudados.

\section{DISCUSSÃO}

Conforme apontamento anterior, os manuais de testes devem informar o usuário a respeito de todas as informações pertinentes à aplicação, à avaliação e à interpretação dos resultados, a fim de que sua utilização atenda aos objetivos propostos.

Não obstante, ao contrário do que se esperava, os resultados desta pesquisa não foram animadores, considerando-se que na amostra estudada foram encontrados instrumentos que não contemplavam os quatro itens que compunham os dados de identificação, ou seja, as informações mais básicas quando se pensa em qualquer publicação científica (nome, autor, editor e data de publicação), embora metade dos testes estudados tenha apresentado todos os itens dos aspectos avaliados.A data de publicação dos instrumentos foi o elemento menos presente.

No que diz respeito aos dados de aplicação e de avaliação, dos onze elementos avaliados, três não estavam presentes na totalidade dos testes psicológicos investigados. De uma maneira geral a análise pertinente à aplicação e à avaliação recebeu uma pontuação maior do que os dados de identificação, exceto no que se refere à bibliografia, que foi o aspecto com menor pontuação. Em relação aos dados referentes às qualidades psicométricas, vale destacar que não foi analisada a consistência ou adequação dos estudos desenvolvidos, registrando-se apenas se houve alguma realização desta natureza.

Os resultados contradizem perspectivas atuais que atestam a extrema relevância de que os instrumentos psicológicos venham acompanhados de materiais com informações suficientes para um uso adequado (Anastasi \& Urbina, 2000; Almeida, 1999; ITC, 2001).

Embora estudos desta natureza sejam necessários, não são freqüentes. Um destaque deve ser dado ao trabalho de Prieto e Muñiz (2000), que elaboraram o questionário que serviu de base para o presente estudo e que se destina a avaliar a qualidade dos instrumentos psicológicos. Os autores acreditam que para que os 
dados obtidos através das aplicações de testes possam ajudar os psicólogos a tomar decisões adequadas, o manual deve reunir as propriedades técnicas oportunas. Para que isto ocorra, os usuários devem receber todas as informações possíveis a respeito das qualidades psicométricas e das características do material.

De uma maneira geral, os instrumentos tiveram avaliações semelhantes, com uma ou outra ressalva apontada ao longo da sessão de resultados. Seria útil que outros estudos desta natureza fossem realizados, como os relacionados à avaliação das características psicométricas dos instrumentos, a fim de que a comunidade psicológica escolhesse os instrumentos com base em dados atuais de pesquisa.

Quanto aos objetivos deste trabalho, acredita-se tê-los atingido, uma vez que os dados revelaram a presença ou ausência de algumas informações essenciais para aplicar, pontuar e avaliar o teste.

\section{BIBLIOGRAFIA}

Adams, K. M. (2000) Practical and Ethical Issues Pertaining to Test Revisions. Psychological Assessment, 12 (3), 281-286.

Almeida, L. S. (1999) Avaliação Psicológica: exigências e desenvolvimentos nos seus métodos. Em: S. M. Wechsler \& R. S. L. Guzzo (Orgs.), Avaliação Psicológica: Perspectiva Internacional.( pp. 41-55) São Paulo: Casa do Psicólogo.

Anastasi , A. \& Urbina, S. (2000) Testagem Psicológica. Porto Alegre: Artes Médicas.

ITC - Comisión Internacional de Tests (2001) Diretrices Internacionales para el uso de los tests. Colegio Oficial de Psicólogos. Disponível em: <http://www.cop.es/tests/Diretrices.htm> (Acessado em: $05 / 05 / 01)$
Cunha, J. A. (2000) Catálogo de Técnicas Úteis. Em: J. A. Cunha e cols. Psicodiagnóstico V. (pp. 202-290) Porto Alegre: Artes Médicas.

Geisinger, K. F. (2000) Psychological Testing at the End of the Millenium: a brief historical review. Professional Psychology: research and practice, 31(2), 117-118.

Okazaki, S. \& Sue, S. (2000) Implications of Test Revisions for Assessment with Asian Americans. Psychological Assessment, 12(3), 272-280.

Prieto, G. \& Muñiz, J. (2000) Um modelo para evaluar la calidad de los tests utilizados em España. Disponível em:<http://www.cop.es/tests/modelo.htm> (Acessado em: 04/12/01)

Salvia, J. \& Ysseldyke, J. (1991) Avaliação em Educação Especial e Corretiva. São Paulo: Manole

Sbardelini, E. T. B. (2001) Avaliação Psicológica e Orientação Profissional. Em Sisto, Sbardelini \& Primi (Orgs.) Contextos e Questões da Avaliação Psicológica. São Paulo: Casa do Psicólogo

Silverstein, M. L. \& Nelson, L. D. (2000) Clinical and Research Implications of Revising Psychological Tests. Psychological Assessment, 12(3), 298-303.

Sisto, F. F.; Sbardelini, E. T. B. \& Primi, R. (Orgs.) (2001) Contextos e Questões da Avaliação Psicológica. (pp. 5-10) São Paulo: Casa do Psicólogo.

Strauss, E.; Spreen, O. \& Hunter, M. (2000) Implications of Test Revisions for Research. Psychological Assessment, 12(3), 237-244.

Wechsler, S. M. (1999) Guia de Procedimentos Éticos para a Avaliação Psicológica. Em S. M. Wechsler \& R. S. L. Guzzo (Orgs.), Avaliação Psicológica: perspectiva internacional. (pp. 133-141) São Paulo: Casa do Psicólogo.

Recebido em 03/09/2001

Revisado em 20/10/2001 Aceito em 30/10/2001 